\title{
Primary Biliary Cirrhosis Associated with Painless Thyroiditis
}

\author{
Naoto Sato, Hiromasa Ohira, Hiroshi Orikasa, Jotaro Shinzawa, Tomohiro Suzuki, Jun Tojo, \\ Masayuki Miyata, Tomoe Nishimaki and Reiji KasuKawa
}

\begin{abstract}
A case of anti-mitochondrial antibody (AMA)-negative primary biliary cirrhosis (PBC) associated with painless thyroiditis is reported in a 47-year-old woman who diagnosed as PBC based on her elevated serum $\gamma$-glutamyl transpeptidase and immunoglobulin $M$ levels, as well as histological findings of destroyed bile ducts surrounded by mononuclear infiltrates in the biopsied liver. She was negative for AMA and had a depressed level of thyroid-stimulating hormone accompanied by increased free thyrosine, thyroxine and triiodothyronine levels and low titers of anti-microsomal and anti-thyroid peroxidase antibodies. Her thyroid disorder corresponded with painless thyroiditis. An association between PBC and hyperthyroidism is rare. Furthermore, an association between AMA-negative PBC and hyperthyroidism due to painless thyroiditis has not previously been reported.
\end{abstract}

(Internal Medicine 38: 244-248, 1999)

Key words: anti-mitochodrial antibody, autoimmune choalangitis, thyroid hormone, hyperthyroidism

\section{Introduction}

Primary biliary cirrhosis (PBC) is an idiopathic liver disease characterized by progressive destruction of the intrahepatic bile ducts. This disease is believed to be caused by autoimmune reactions (1). The autoimmune etiology for $\mathrm{PBC}$ is supported by its frequent association with other autoimmune diseases such as Sjögren's syndrome, rheumatoid arthritis, Hashimoto's thyroiditis, and CREST syndrome (2). Although thyroid dysfunction is the most common nonhepatic lesion associated with $\mathrm{PBC}$, hyperthyroidism is rarely associated with $\operatorname{PBC}(2,3)$. Furthermore, an association between hyperthyroidism due to painless thyroiditis and $\mathrm{PBC}$ has not previously been reported.

Recently, it has been recognized that $5-10 \%$ of PBC patients are negative for the anti-mitochondrial antibody (AMA) with similar clinical, histological, and other laboratory features as AMA-positive $\operatorname{PBC}(1,4)$. On the other hand, some forms of AMA-negative PBC are believed to be a distinct clinical entity designated as autoimmune cholangitis (5-7). However, it is suggested that AMA-positive and AMA-negative PBC share a similar clinical spectrum and outcome $(8,9)$. The present paper describes a patient with AMA-negative PBC associated with hyperthyroidism caused by painless thyroiditis.

\section{Case Report}

A 47-year-old woman was admitted to our department in February 1996 due to liver dysfunction. She was not an alcoholic and had no history of blood transfusion or drug allergy. She had been suffering from pruritus for five years. Two years earlier, she had been found to have an elevated level of $\gamma$ glutamyl transpeptidase $(\gamma-\mathrm{GTP})$ at $361 \mathrm{IU} / \mathrm{l}$ (normal range, 630). In November 1995, she had noticed a finger tremor and her $\gamma$-GTP level was elevated at $232 \mathrm{IU} / l$. Her status on admission was as follows: height, $156.0 \mathrm{~cm}$; body weight, $53.6 \mathrm{~kg}$; blood pressure, 124/80 mmHg; pulse, regular at 92 beats $/ \mathrm{min}$. The skin on her back was slightly pigmented and scratched. No goiter nor enlargement of the cervical lymph nodes was noted. No remarkable abnormal changes were noted in her heart of lungs. The liver edge was palpable one fingerbreadth under the right costal edge without tenderness. Laboratory data on admission are shown in Table 1. A moderate increase in alkaline phosphatase (ALP), $574 \mathrm{IU} / l$; with ALP2 isozyme 81\%, $\gamma$ GTP, $210 \mathrm{IU} / l$; and leucine aminopeptidase (LAP), 177 IU/l was noted. Serum immunoglobulin M (IgM) level was elevated to $746 \mathrm{mg} / \mathrm{dl}$. AMA was not detectable by indirect immunofluorescence nor immunoblotting using pyruvate dehydrogenase complex taken from bovine heart mitochondria as described previously (10). She was positive for antinuclear anti-

From the Department of Internal Medicine II, Fukushima Medical University School of Medicine, Fukushima

Received for publication May 18, 1998; Accepted for publication November 16, 1998

Reprint requests should be adressed to Dr. Hiromasa Ohira, the Department of Internal Medicine II, Fukushima Medical University School of Medicine, 1 Hikarigaoka, Fukushima 960-1295 
Table 1. Laboratory Data on Admission

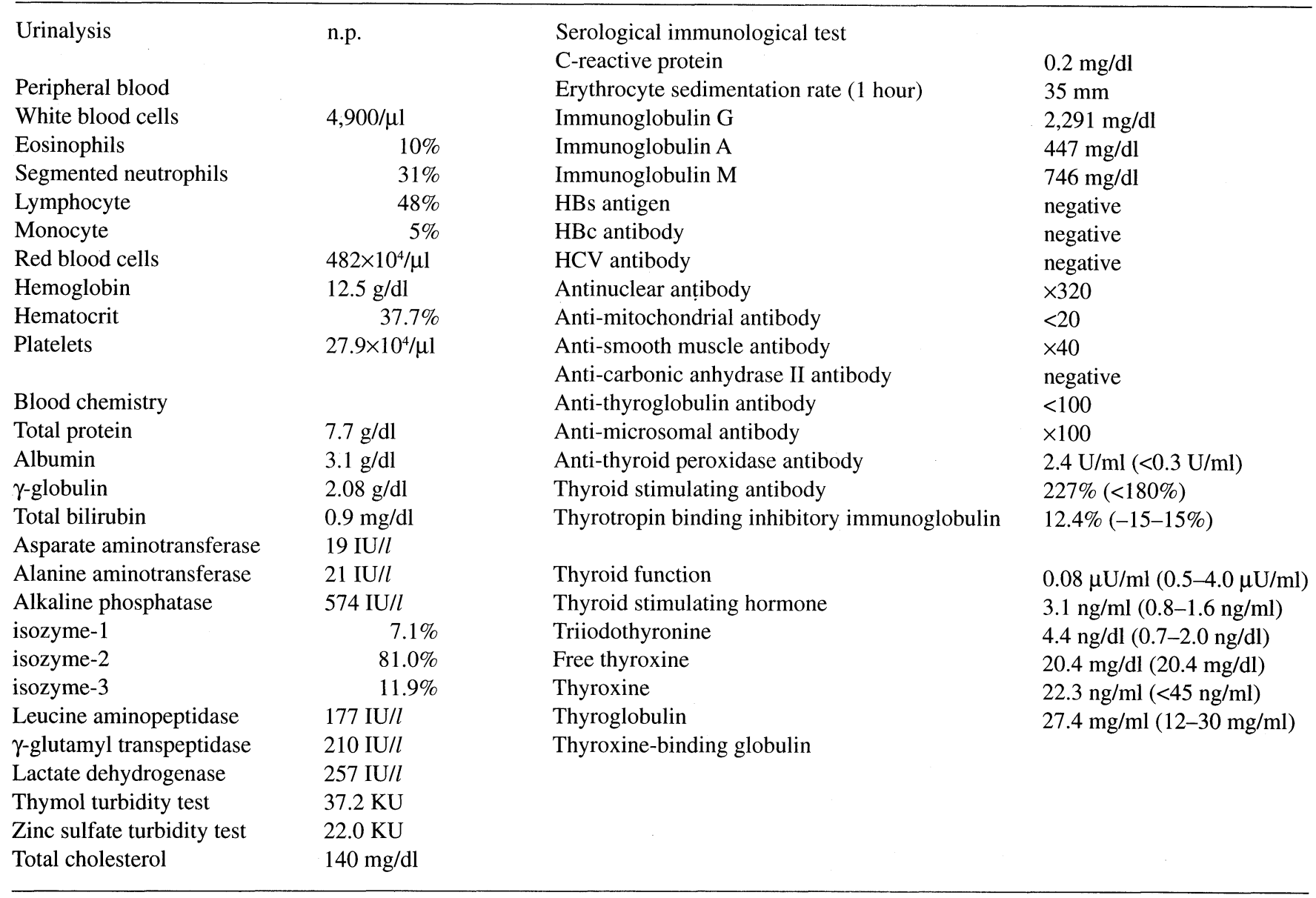

HBsAg: hepatitis B virus surface antigen, APTT: activated partial thromboplastin time, AST: aspartate aminotransferase, ALT: alanine aminotransferase, vWF: von Willebrand factor.

body (ANA, 1:320) and anti-smooth muscle antibody (ASMA, 1:40). However, she was negative for antibody against carbonic anhydrase II (Sigma Chemical Co., St. Louis, MO) according to Western blotting (11). Tests for hepatitis B virus surface (HBs)-antigen, hepatitis B virus core antigen (HBc)-antibody and hepatitis $\mathrm{C}$ virus (HCV)-antibody were all negative.

Thyroid function tests showed increased levels of free thyroxine (T4) $4.4 \mathrm{ng} / \mathrm{dl}, \mathrm{T} 420.4 \mu \mathrm{g} / \mathrm{dl}$ and triiodothyronine (T3) $3.1 \mathrm{ng} / \mathrm{dl}$ as well as a depressed thyroid-stimulating hormone (TSH) level of $0.08 \mu \mathrm{U} / \mathrm{ml}$. Low titers of anti-microsomal (MCHA) and anti-thyroid peroxidase antibody (TPOAb) were noted. Anti-thyrogulobulin (TGHA) was not detected. AntiTSH receptor antibodies (TBII) and thyroid stimulating antibody (TSAb) levels were normal as shown in Table 1. A thyroid scan using ${ }^{123}$ I revealed no increased uptake in the thyroid area. These findings suggested a diagnosis of painless thyroiditis. She was treated with $150 \mathrm{mg} /$ day of thiamazole. Her thyroid function became normal 2 months later and TSH returned to normal range 6 months later (Fig. 1).

Abdominal ultrasonography and computed tomography showed no evidence of biliary dilatation or gallbladder dis- ease. Endoscopic cholangiography revealed normal intrahepatic and extrahepatic bile ducts. The histology of the biospied liver showed destruction of several bile ducts surrounded by a moderate infiltration of mononuclear cells and by granuloma with epithelioid cells in the portal area as seen in Fig. 2. Based on the clinical criteria for diagnosing $\mathrm{PBC}$ and also on the histologic findings, she was diagnosed as AMA-negative PBC and underwent treatment with ursodeoxycholic acid at $600 \mathrm{mg} /$ day. Six months later, her serum ALP and $\gamma$-GTP levels decreased to $342 \mathrm{IU} / l$ and $77 \mathrm{IU} / l$, respectively (Fig. 1).

\section{Discussion}

Researchers in the early 1980s reported a higher incidence of thyroid dysfunction in patients with $\operatorname{PBC}(12,13)$. Although the hormone levels in the thyroid are reportedly lower in 12$18 \%$ of PBC patients (3), hyperthyroidism associated with PBC has only been reported in four patients: three with Graves' disease and one with Hashitoxicosis (14-17). There have been no previous reports of painless thyroiditis associated with PBC.

Painless thyroiditis was first reported by Hamberger in 1971 


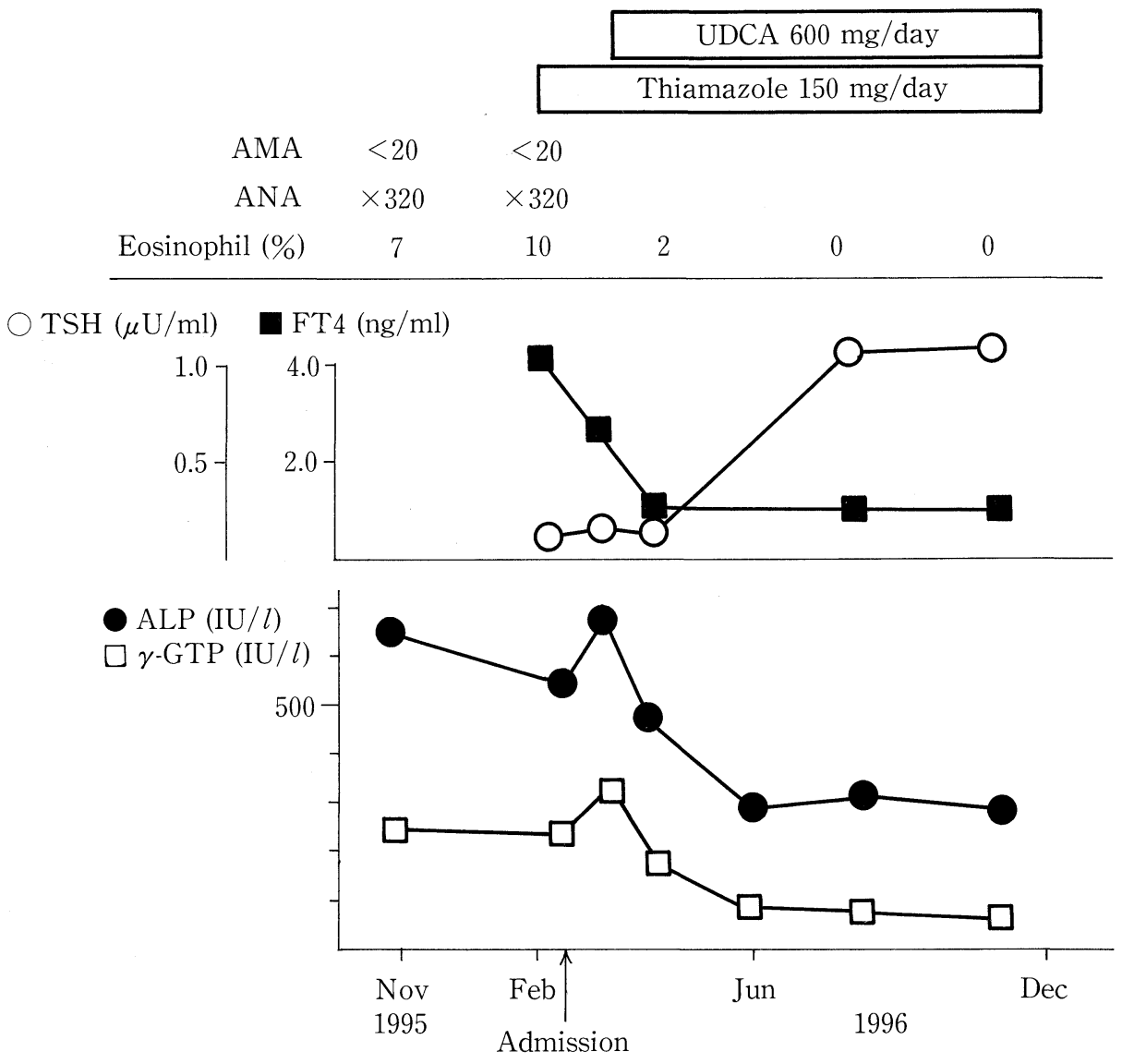

Figure 1. Clinical course of the patient. UDCA: ursodeoxycholic acid.

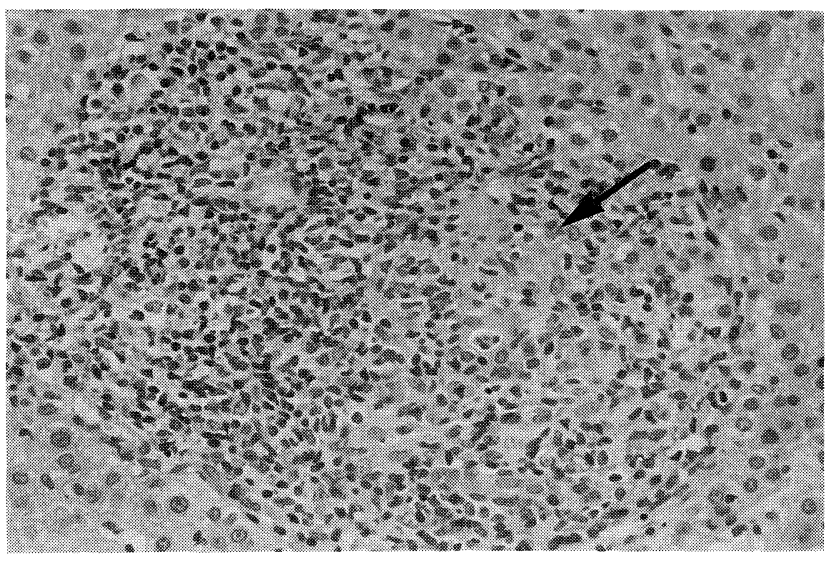

Figure 2. Histological findings of biopsied liver specimen taken in February 1996. Moderate infiltration of mononuclear cells with a few eosinophils and destructed small bile ducts in the enlarged portal area were observed. An epithelioid granuloma (arrow) contacted with a damaged small bile duct. The intensity of both ductural proliferation and fibrosis was slight. These findings showed PBC at early stage (Scheuer' stage 1) (HE stain, $\times 250$ ). as a transient form of destructive thyrotoxicosis designated as occult subacute thyroiditis (18). This disease is histologically characterized by a nontender goiter, depressed thyroidal radioiodine uptake, negative or low titers of antithyroid antibodies, and lymphocytic thyroiditis (19). The thyrotoxicosis present in this disease is different from that of Graves' disease, because intrathyroidal hormones leak through the thyroidal epithelial cells damaged by autoimmune inflammation. This condition also differs from subacute thyroiditis in that there is no tenderness of the thyroid, and no marked elevation in the erythrocyte sedimentation rate and C-reactive protein level. Another difference is the presence of autoantibodies to the thyroid gland. As a result, painless thyroiditis was considered to be a subacute aggravated form of Hashimoto's disease or autoimmune thyroiditis (19). However, patients with Hashimoto's disease often demonstrate markedly elevated anti-thyroid antibody levels and differ histologically in some respects from painless thyroiditis patients. Nagataki reported that painless thyroiditis could be classified as an antoimmune thyroiditis, separate from Hashimoto's disease (20). In the present report, the condition was diagnosed as painless thyroiditis on the basis of thyrotoxicosis with a low radioiodine uptake, low levels of MCHA and TPOAb, and normal levels of TBII and TSAb.

The etiology of painless thyroiditis remains unclear. The 
importance of environmental factors or autoantigens has been suggested $(21,22)$. The present patient had no episodes of viral infection, demonstrated no evidence of circulating autoantibodies and had not been administered immunosupressants. The seasonal recurrence of painless thyroiditis suggests an allergic component to this disease as proposed by Yamamoto et al (23). Eosinophilia and eosinophilic infiltration of the liver have often been found in patients with $\operatorname{PBC}(24,25)$. In the present case, eosinophilia $(490 / \mu \mathrm{l})$ was also noted and it would be of interest to consider a relationship between eosinophilia and painless thyroiditis.

The disease of $\mathrm{PBC}$ negative for AMA and positive for ANA has been considered as a distinct disease and designated as autoimmune cholangitis (5-7). Whether autoimmune cholangitis is an independent entity or simple PBC without the serum AMA remains under discussion. Some authors have found differential features, such as piecemeal necrosis, positive ASMA, higher serum aminotransferase levels, lower serum IgM levels, and a benefical effect of prednisone and azathioprine in patients with autoimmune cholangitis (26). On the other hand, a similar clinical spectrum and a similar outcome of the disease have been reported in AMA-negative and AMA-positive $\mathrm{PBC}$, although different patterns of immunoreactivity are present in the two conditions $(8,9)$. In the case presented here, normal serum aminotransferase levels and higher serum IgM levels were noted and serum ALP and $\gamma$-GTP levels were improved with ursodeoxycholic acid. Therefore it would be appropriate to diagnose this as AMA-negative PBC. Recently, an association between the presence of anti-human carbonic anhydrase II and autoimmune cholangitis has been reported, although this has been observed in only a small number of patients (11). Carbonic anhydrase II, a zinc metal enzyme, is present in the epithelium of bile ducts in the liver, but not in hepatocytes (27). The physiological function of carbonic anhydrase II in the liver has not yet been elucidated. Pain et al reported that the carbonic anhydrase II gene is transcriptionally activated by the thyroid hormone T3 (28). Thus, the presence of the anti-carbonic anhydrase II antibody and thyroid dysfunction should be considered in AMA-negative PBC, although the present patient was negative for the anti-carbonic anhydrase II antibody.

The present patient represents the first reported case of AMAnegative $\mathrm{PBC}$ associated with painless thyroiditis. The mechanism of association between these two diseases is not clear. However, it appears to involve an autoimmune component. The present patient may represent a combined variant of $\mathrm{PBC}$ and Hashimoto's disease.

\section{References}

1) Kaplan MM. Primary biliary cirrhosis. N Eng J Med 316: 521-528, 1987.

2) Heathcote $\mathbf{J}$. The clinical expression of primary biliary cirrhosis. Semin Liver Dis 17: 23-33, 1997.

3) Huang MJ, Liaw YF. Clinical associations between thyroid and liver diseases. J Gastroenterol Hepatol 10: 344-350, 1995.

4) Gershwin ME, Mackay IR. Primary biliary cirrhosis: paradigm or paradox for autoimmunity. Gastroenterology 100: 822-833, 1991.
5) Brunner G, Klinge O. A chronic destructive non-suppurative cholangitislike disease picture with antinuclear antibodies (immunocholangitis). Dtsch Med Wochenschr 112: 1454-1458, 1987.

6) Ben-Ari Z, Dhillon AP, Sherlock S. Autoimmune cholangiopathy: part of the spectrum of autoimmune chronic active hepatitis. Hepatology 18: 1015, 1993.

7) Michieletti P, Wanless IR, Katz A, et al. Antimitochondrial antibody-negative primary biliary cirrhosis: a distinct syndrome of antoimmune cholangitis. Gut 35: 260-265, 1994.

8) Goodman ZD, Mcnally PR, Davis DR, Ishak KG. Autoimmune cholangitis: a variant of primary biliary cirrhosis. Clinicopathologic and serologic correlations in 200 cases. Dig Dis Sci 40: 1232-1242, 1995.

9) Invernizzi P, Crosignani A, Battezzati PM, et al. Compatison of the clinical features and clinical course of antimitochondrial antibody-positive and -negative primary biliary cirrhosis. Hepatology 25: 1090-1095, 1997.

10) Kuroda M, Morito T, Takagi T, et al. Antibodies to E1 and E2/protein $X$ components of pyruvate dehydrogenase complex in sera of patients with primary biliary cirrhosis. J Hepatol 25: 867-876, 1996.

11) Gordon SC, Quattrociocchi-Longe TM, Khan BA, et al. Antibodies to carbonic anhydrase in patients with immune cholangiopathies. Gastroenterology 108: 1802-1809, 1995.

12) Crowe JP, Christensen E, Butler J, et al. Primary Biliary cirrhosis: the prevalence of hypothyroidism and its relationship to thyroid autoantibodies and sicca syndrome. Gastroenterology 78: 1437-1441, 1980.

13) Elta GH, Sepersky RA, Goldberg MJ, Connors CM, Miller KB, Kaplan MM. Increased incidence of hypothyroidism in primary biliary cirrhosis. Dig Dis Sci 28: 971-975, 1982.

14) Nieri S, Riccardo GG, Salvadori G, Surrenti C. Primary biliary cirrhosis and Graves' disease. J Clin Gastroentero 7: 434-437, 1985.

15) Sasaki H, Matake H, Eimoto T, Shimizu M, Aoyama S, Okumura M. Asymptomatic primary biliary cirrhosis (A-PBC) associated with so-called "Hashitoxicosis" (in Japanese). Nippon Naika Gakkai Zasshi 76: 746747, 1987.

16) Thompson NP, Leader S, Jamieson CP, Burnham WR, Burroughs AK. Reversible jaundice in primary biliary cirrhosis due to hyperthyroidism. Gastroenterology 106: 1342-1343, 1994.

17) Tateishi M, Hara Y, Sakurai T. A case of mixed connective tissue disease associated with Graves' disease and primary biliary cirrhosis. Jpn J Clin Immun 17: 611-616, 1994 (in Japanese).

18) Hamburger JI. Occult subacute thyroiditis-diagnostic challenge. Mich Med 70: 1125-1227, 1971.

19) Amino N. Postpartum and silent thyroiditis. in: Thyroid Diseases Vol II. Clinical Fundamental and Therapy, Monaco F, Satta MA, Shapiro B, Troncone L, Eds. CRC Press Inc., Boca Raton, FL, USA, 1993, pp. 239249.

20) Nagataki S. The concept of Hashimoto's disease. in: 80 Years of Hashimoto's Disease. Nagataki S, Mori T, Torizuka K, Eds. Elsevier Science, Amsterdam, 1993, p. 539.

21) Schneeberg NG. Silent thyroiditis. Arch Intern Med 143: 2214, 1983 (letter).

22) Jenkins H, Farid NR. Subacute thyroiditis-like syndromes -Relation to HLA. Tissue Antigens 13: 167-169, 1979.

23) Yamamoto M, Shibuya N, Chen LC, Ogata E. Seasonal recurrence of transient hypothyroidism in a patient with autoimmune thyroiditis. Endocrinol Jpn 35: 135-142, 1988.

24) Kokubun M, Kuroda M, Takagi T, et al. Eosinophilia in primary biliary cirrhosis. Nippon Shokakibyo Gakkai Zasshi 87: 1410-1416, 1990 (in Japanese).

25) Terasaki S, Nakanuma Y, Yamazaki M, Unoura M. Eosinophilic infiltration of the liver in primary biliary cirrhosis: A morphological study. Hepatology 17: 206-212, 1993.

26) Sànchez-Pobre $P$, Castellano $G$, Colina $F$, et al. Antimitochondrial antibody-negative chronic nonsuppurative destractive cholangitis. Atypical primary biliary cirrhosis or autoimmune cholangitis? J Clin Gastroenterol 23: 191-198, 1996.

27) Spicer SS, Sens MA, Tashian RE. Immunohistochemical demonstration of carbonic anhydrase in human epithelial cells. J Histochem Cytochem 


\section{SATO et al}

30: $864-873,1982$.

28) Pain B, Melet F, Jurdic P, Samarut J. The carbonic anhydrase II gene, a gene regulated by thyroid hormone and erythropoietin, is repressed by the v-erbA oncogene in erythrocytic cells. New Biol 2: 284-294, 1990. 